\title{
A New Compensation Method for Measurement of Thread Pitch Diameter by Profile Scanning
}

\author{
Chen Sheng, Zhao Dongbiao, Lu Yonghua \\ College of Mechanical and Electrical Engineering, Nanjing University of Aeronautics and Astronautics, No.29 Yudao \\ Street, 210016, Nanjing, China, e-mail: chensheng351@qq.com
}

\begin{abstract}
For measurement of existing thread parameters using profile scanning, system error increases as pitch diameter, difference between the calibrating thread and measuring thread increases with the single gauge calibration method. There are several alignment deviations affecting the measurement of pitch diameter. To reduce the system error, a compensation method using two standard thread gauges to calculate the alignment deviations is proposed here. Finally, the theoretical and experimental comparisons between the single gauge calibration method and the proposed method are presented. The results demonstrate that the two gauges calibration method can effectively reduce the system error.
\end{abstract}

Keywords: Thread measurement, pitch diameter, compensation, two gauges calibration.

\section{INTRODUCTION}

$\mathrm{A}$ MONG VARIOUS thread parameters, the pitch diameter is the most commonly used criterion for thread measurement. Almost all the methods are committed to improving the measurement accuracy of the pitch diameter. Classical methods and procedures for calibrating the pitch diameter of a thread ring, such as the two balls method, are executed by means of universal measuring machines or using coordinate measuring machines [1]. Modern methods, which deal with scanning the profile and mathematical processing of the received data, achieve improvement in the detection efficiency by calculating the multiple parameters of the thread ring simultaneously [2].

Kosarevsky [3] utilized the general Hough transformation to deal with the thread profile data and obtained the intersections of each adjacent segment pair, which can identify the circles tangent with the profile segments. Then, the pitch diameter can be calculated based on the Berndt formula [4]. This approach is an extension of the two balls method actually. The accuracy of the pitch diameter result depends on the measurement accuracy of other parameters such as pitch, flank angle, and diameter of the balls. Hong [5] studied two mainly non-contact methods: one uses a CCD camera with an attached sight pipe illumination unit and the other uses a laser sensor and a motorized periscope. Huang [6] and Tong [7] achieved good accuracy for the pitch diameter results with the laser triangulation scanning method. However, the influence on the pitch diameter caused by the alignment deviations has not been comprehensively analyzed. Shchurov [8] and Kosarevsky [9] provided the calculation approach of the pitch diameter from the $3 \mathrm{D}$ datasets, which are obtained by the CMM and the CT scanner, respectively. The MasterScanner series thread measurement instruments, developed by the IAC Corporation, can present the pitch diameter result with high traceability. According to the published patents and articles $[10,11]$, the calculation procedure of the pitch diameter is based on the combination of the top diameter and the distance between the pitch diameter line and the top diameter line. The single gauge calibration method is adopted to compensate the error caused by the alignment deviations. A simplified compensation model is used in this case and high precision of alignment is required to guarantee measurement accuracy of the pitch diameter.

The purpose of this work is to analyze the effect of alignment deviations on pitch diameter and propose a compensation method to calibrate these alignment deviations. The present work has been organized as follows. In section 1, the measurement principle is introduced. Section 2 analyzes the effect of alignment deviations on pitch diameter caused by both the probe and work piece. Then, the compensation method using a two gauges calibration is discussed in section 3. Sections 4 and 5 present the test results and conclusion, respectively.

\section{MEASUREMENT PRINCIPLE}

The structure of the $2 \mathrm{D}$ thread measurement instrument with tactile scanning developed in our laboratory is shown in Fig.1.

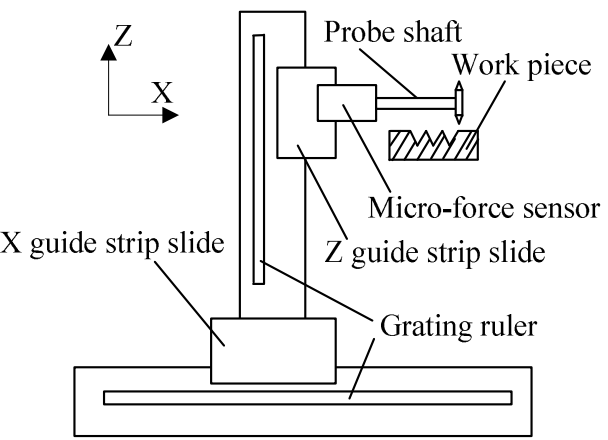

Fig.1. Structure of the 2D thread measuring instrument.

Based on the aerostatic slideway, the motion platform is made of a guide strip slide structure, which is driven by the linear motor. The position feedback is implemented by a grating ruler. During the measurement, the $X$ axis platform moves at a constant velocity, whereas the $Z$ axis platform 
follows the contour curve with constant contact force controlled by the micro-force sensor feedback. The contour data are collected and processed after the scanning. Thread parameters such as pitch diameter, flank angle and pitch can be calculated from these contour data.

\section{THE INFLUENCE OF THE ALIGNMENT DEVIATIONS}

The homogeneous transformation method is usually used to build the volumetric error model [12]. In order to express an arbitrary point in the whole workspace of the measuring instrument, we construct a coordinate system OXYZ, the origin of which is fixed at the initial point of the instrument. Besides, the coordinate system $\mathrm{O}_{\mathrm{W}} \mathrm{X}_{\mathrm{W}} \mathrm{Y}_{\mathrm{W}} \mathrm{Z}_{\mathrm{W}}$ is set to describe the geometry model of the work piece. The work pieces are all axisymmetric bodies, no matter whether they are cylindrical or conical shapes. Consequently, the origin of the coordinate system $\mathrm{O}_{\mathrm{W}} \mathrm{X}_{\mathrm{W}} \mathrm{Y}_{\mathrm{W}} \mathrm{Z}_{\mathrm{W}}$ can be fixed at the central point on the end face of the work piece. According to the definition of the machine coordinate, the space coordinates of the system can be defined as shown in Fig.2.

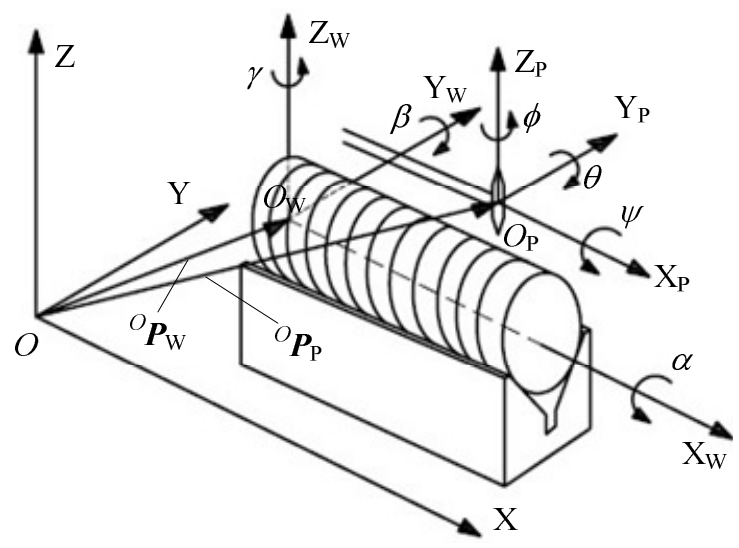

Fig.2. Coordinate system definition.

To facilitate the display of the coordinate system, the alignment method shown in Fig.2. is the V-block location method, which is used for external threads. Internal threads with different location fixtures have a similar formula inference. The coordinate system OXYZ in Fig.2. is the basic coordinate system of the instrument. $\mathrm{O}_{\mathrm{W}} \mathrm{X}_{\mathrm{W}} \mathrm{Y}_{\mathrm{W}} \mathrm{Z}_{\mathrm{W}}$ is the coordinate system for the work piece and $\mathrm{O}_{\mathrm{P}} \mathrm{X}_{\mathrm{P}} \mathrm{Y}_{\mathrm{P}} \mathrm{Z}_{\mathrm{P}}$ represents the probe coordinate system. The coordinate system $\mathrm{O}_{\mathrm{W}} \mathrm{X}_{\mathrm{W}} \mathrm{Y}_{\mathrm{W}} \mathrm{Z}_{\mathrm{W}}$ can be obtained through the.shifting transformation ${ }^{\mathrm{O}} \boldsymbol{P}_{\mathrm{W}}\left(a_{\mathrm{W}}, b_{\mathrm{W}}, c_{\mathrm{W}}\right)$ and rotating transformation $(\alpha, \beta, \gamma)$ from the coordinate system OXYZ. The homogeneous transformation matrix can be expressed as follows:

$$
{ }_{\mathrm{W}}^{\mathrm{O}} \boldsymbol{T}=\boldsymbol{T r a n s}\left(a_{\mathrm{W}}, b_{\mathrm{W}}, c_{\mathrm{W}}\right) \boldsymbol{R o t}(x, \alpha) \boldsymbol{R o t}(y, \beta) \boldsymbol{R o t}(z, \gamma)
$$

The pitch diameter is an ideal cylinder diameter, so the work piece can be regarded as an axisymmetric body around the $\mathrm{X}$ axis. With the definition of coordinate system, rotation angle $\alpha$ around the $\mathrm{X}$ axis can be equal to 0 . Therefore, rotation transformation $\boldsymbol{R o t}(x, \alpha)$ turns into the unit matrix, formula (1) can be simplified as follows:

$$
\begin{aligned}
& { }_{\mathrm{W}}^{\mathrm{T}} \boldsymbol{T}=\boldsymbol{T} \boldsymbol{\operatorname { r a n s }}\left(a_{\mathrm{W}}, b_{\mathrm{W}}, c_{\mathrm{W}}\right) \boldsymbol{R o t}(y, \beta) \boldsymbol{R o t}(z, \gamma)= \\
& {\left[\begin{array}{cccc}
1 & 0 & 0 & a_{\mathrm{W}} \\
0 & 1 & 0 & b_{\mathrm{W}} \\
0 & 0 & 1 & c_{\mathrm{W}} \\
0 & 0 & 0 & 1
\end{array}\right]\left[\begin{array}{cccc}
\mathrm{c} \beta & 0 & \mathrm{~s} \beta & 0 \\
0 & 1 & 0 & 0 \\
-\mathrm{s} \beta & 0 & \mathrm{c} \beta & 0 \\
0 & 0 & 0 & 1
\end{array}\right]\left[\begin{array}{cccc}
\mathrm{c} \gamma & -\mathrm{s} \gamma & 0 & 0 \\
\mathrm{~s} \gamma & \mathrm{c} \gamma & 0 & 0 \\
0 & 0 & 1 & 0 \\
0 & 0 & 0 & 1
\end{array}\right]} \\
& =\left[\begin{array}{cccc}
\mathrm{c} \beta \mathrm{c} \gamma & -\mathrm{c} \beta \mathrm{s} \gamma & \mathrm{s} \beta & a_{\mathrm{W}} \\
\mathrm{s} \gamma & \mathrm{c} \gamma & 0 & b_{\mathrm{W}} \\
-\mathrm{s} \beta \mathrm{c} \gamma & \mathrm{s} \beta \mathrm{s} \gamma & \mathrm{c} \beta & c_{\mathrm{W}} \\
0 & 0 & 0 & 1
\end{array}\right]
\end{aligned}
$$

Similarly, the homogeneous transformation matrix from the coordinate system OXYZ to coordinate system $\mathrm{O}_{\mathrm{P}} \mathrm{X}_{\mathrm{P}} \mathrm{Y}_{\mathrm{P}} \mathrm{Z}_{\mathrm{P}}$ can be obtained as follows:

$$
{ }_{\mathrm{P}}^{\mathrm{O}} \boldsymbol{T}=\boldsymbol{T r a n s}\left(a_{\mathrm{P}}, b_{\mathrm{P}}, c_{\mathrm{P}}\right) \boldsymbol{R o t}(x, \psi) \boldsymbol{R o t}(y, \theta) \boldsymbol{R o t}(z, \phi)
$$

Considering the probe is an axisymmetric body around the $\mathrm{Z}$ axis, we then let the origin of the probe coordinate system $\mathrm{O}_{\mathrm{P}} \mathrm{X}_{\mathrm{P}} \mathrm{Y}_{\mathrm{P}} \mathrm{Z}_{\mathrm{P}}$ be in the middle of the central line so that $\phi=0$ with the definition of coordinate system. Therefore, rotation transformation $\boldsymbol{R} \boldsymbol{\operatorname { t o t }}(z, \phi)$ turns into the unit matrix, formula (3) can be simplified as follows:

$$
\begin{aligned}
& { }_{\mathrm{P}}^{\mathrm{T}} \boldsymbol{T}=\boldsymbol{T r a n s}\left(a_{\mathrm{P}}, b_{\mathrm{P}}, c_{\mathrm{P}}\right) \boldsymbol{R o t}(x, \psi) \boldsymbol{R o t}(y, \theta)= \\
& {\left[\begin{array}{cccc}
1 & 0 & 0 & a_{\mathrm{P}} \\
0 & 1 & 0 & b_{\mathrm{P}} \\
0 & 0 & 1 & c_{\mathrm{P}} \\
0 & 0 & 0 & 1
\end{array}\right]\left[\begin{array}{cccc}
1 & 0 & 0 & 0 \\
0 & \mathrm{c} \psi & -\mathrm{s} \psi & 0 \\
0 & \mathrm{~s} \psi & \mathrm{c} \psi & 0 \\
0 & 0 & 0 & 1
\end{array}\right]\left[\begin{array}{cccc}
\mathrm{c} \theta & 0 & \mathrm{~s} \theta & 0 \\
0 & 1 & 0 & 0 \\
-\mathrm{s} \theta & 0 & \mathrm{c} \theta & 0 \\
0 & 0 & 0 & 1
\end{array}\right]} \\
& =\left[\begin{array}{cccc}
\mathrm{c} \theta & 0 & \mathrm{~s} \theta & a_{\mathrm{P}} \\
\mathrm{s} \psi \mathrm{s} \theta & \mathrm{c} \psi & -\mathrm{s} \psi \mathrm{c} \theta & b_{\mathrm{P}} \\
-\mathrm{c} \psi \mathrm{s} \theta & \mathrm{s} \psi & \mathrm{c} \psi \mathrm{c} \theta & c_{\mathrm{P}} \\
0 & 0 & 0 & 1
\end{array}\right]
\end{aligned}
$$

Assume that the probe height is $h_{\mathrm{P}}$, so the coordinates of both upper and downward tip points in the OXYZ system can be described as follows:

$$
\begin{aligned}
& { }^{\mathrm{O}} \boldsymbol{P}^{\text {up }}={ }_{\mathrm{P}}^{\mathrm{O}} \boldsymbol{T}^{\mathrm{P}} \boldsymbol{P}^{\text {up }}= \\
& {\left[\begin{array}{cccc}
\mathrm{c} \theta & 0 & \mathrm{~s} \theta & a_{\mathrm{P}} \\
\mathrm{s} \psi \mathrm{s} \theta & \mathrm{c} \psi & -\mathrm{s} \psi \mathrm{c} \theta & b_{\mathrm{P}} \\
-\mathrm{c} \psi \mathrm{s} \theta & \mathrm{s} \psi & \mathrm{c} \psi \mathrm{c} \theta & c_{\mathrm{P}} \\
0 & 0 & 0 & 1
\end{array}\right]\left[\begin{array}{c}
0 \\
0 \\
\frac{h_{\mathrm{P}}}{2} \\
1
\end{array}\right]=\left[\begin{array}{c}
\frac{h_{\mathrm{P}} \mathrm{s}}{2}+a_{\mathrm{P}} \\
\frac{h_{\mathrm{P}} \mathrm{s} \psi \mathrm{c} \theta}{2}+b_{\mathrm{P}} \\
\frac{h_{\mathrm{P}} \mathrm{c} \psi \mathrm{c} \theta}{2}+c_{\mathrm{P}} \\
1
\end{array}\right]}
\end{aligned}
$$




$$
\begin{aligned}
& { }^{\mathrm{O}} \boldsymbol{P}^{\mathrm{down}}={ }_{\mathrm{P}}^{\mathrm{O}} \boldsymbol{T}^{\mathrm{P}} \boldsymbol{P}^{\text {down }}= \\
& {\left[\begin{array}{cccc}
\mathrm{c} \theta & 0 & \mathrm{~s} \theta & a_{\mathrm{P}} \\
\mathrm{s} \psi \mathrm{s} \theta & \mathrm{c} \psi & -\mathrm{s} \psi \mathrm{c} \theta & b_{\mathrm{P}} \\
-\mathrm{c} \psi \mathrm{s} \theta & \mathrm{s} \psi & \mathrm{c} \psi \mathrm{c} \theta & c_{\mathrm{P}} \\
0 & 0 & 0 & 1
\end{array}\right]\left[\begin{array}{c}
0 \\
0 \\
-\frac{h_{\mathrm{P}}}{2} \\
1
\end{array}\right]=\left[\begin{array}{c}
-\frac{h_{\mathrm{P}} \mathrm{s} \theta}{2}+a_{\mathrm{P}} \\
\frac{h_{\mathrm{P}} \mathrm{s} \boldsymbol{c} \theta}{2}+b_{\mathrm{P}} \\
2 \\
-\frac{h_{\mathrm{P}} \mathrm{c} \psi \mathrm{c} \theta}{2}+c_{\mathrm{P}} \\
1
\end{array}\right](6)}
\end{aligned}
$$

where ${ }^{\mathrm{P}} \boldsymbol{P}^{\text {up }},{ }^{\mathrm{P}} \boldsymbol{P}^{\text {down }}$ are the coordinate vectors of up and down tip points in the probe coordinate system, respectively. The motion of the probe is restricted in the $\mathrm{XZ}$ plane, the shifting coordinates of the $\mathrm{X}$ axis $a_{\mathrm{P}}$ and the $\mathrm{Z}$ axis $c_{\mathrm{P}}$ change with the movement of the probe. However, the $Y$ axis coordinate of the probe tip points will not change. Hence,

$$
\left\{\begin{array}{l}
{ }^{\mathrm{o}} P_{y}^{\text {up }}=-\frac{h_{\mathrm{P}} \mathrm{s} \psi \mathrm{c} \theta}{2}+b_{\mathrm{P}}=\text { const } 1 \\
{ }^{\mathrm{o}} P_{y}^{\text {down }}=\frac{h_{\mathrm{P}} \mathrm{c} \psi \mathrm{c} \theta}{2}+b_{\mathrm{P}}=\text { const } 2
\end{array}\right.
$$

On the other hand, the abrasion of thread work piece is not uniform. Usually, abrasion is larger near the interface part when compared to other parts. Fig.3. is a sectional view of a ring gauge. As a result of the non-uniform abrasion, the fitted up and down pitch diameter lines turn out to be the cone's generators.

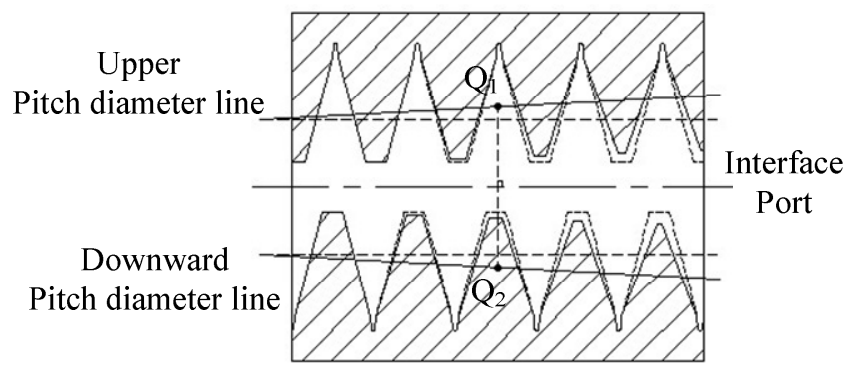

Fig.3. Sectional view of a ring gauge.

To calculate the average value of the thread pitch diameter, we choose the distance between the $\mathrm{Q}_{1}$ and $\mathrm{Q}_{2}$ which are the middle points of the upper and downward pitch diameter line segments, respectively. Assume the coordinates of the points $\mathrm{P}_{1}$ and $\mathrm{P}_{2}$ in the work piece coordinate system are ${ }^{\mathrm{W}} \boldsymbol{Q}_{1}=\left[{ }^{\mathrm{W}} Q_{x 1},{ }^{\mathrm{W}} Q_{y 1},{ }^{\mathrm{W}} Q_{z 1}\right]^{\mathrm{T}},{ }^{\mathrm{W}} \boldsymbol{Q}_{2}=\left[{ }^{\mathrm{W}} Q_{x 2},{ }^{\mathrm{W}} Q_{y 2},{ }^{\mathrm{W}} Q_{z 2}\right]^{\mathrm{T}}$. If the valid length of the thread gauge is $L$, then ${ }^{\mathrm{W}} Q_{x 1}={ }^{\mathrm{W}} Q_{x 2}=L / 2$. The measuring pitch diameter, namely the distance between the points $\mathrm{P}_{1}$ and $\mathrm{P}_{2}$ in the $\mathrm{XZ}$ plane based on the OXYZ coordinate system, can be calculated as follows:

$$
\begin{aligned}
D_{\mathrm{c}}= & \left.\operatorname{dist}\left({ }^{\mathrm{C}} \boldsymbol{Q}_{1},{ }^{\mathrm{o}} \boldsymbol{Q}_{2}\right)\right|_{\mathrm{XZ}}=\left\|{ }^{\mathrm{O}} \boldsymbol{Q}_{1}-\boldsymbol{Q}_{2}\right\|_{\mathrm{Xz}}=\left\|{ }_{\mathrm{W}}^{\mathrm{O}} \boldsymbol{T}^{\mathrm{W}} \boldsymbol{Q}_{1}-{ }_{\mathrm{W}}^{\mathrm{O}} \boldsymbol{T}^{\mathrm{W}} \boldsymbol{Q}_{2}\right\|_{\mathrm{xz}} \\
& =\left\|\left[\begin{array}{c}
\left({ }^{\mathrm{W}} Q_{x 1}-{ }^{\mathrm{W}} Q_{x 2}\right) \mathrm{c} \beta \mathrm{c} \gamma-\left({ }^{\mathrm{W}} Q_{y 1}-{ }^{\mathrm{W}} Q_{y 2}\right) \mathrm{c} \beta \mathrm{s} \gamma+\left({ }^{\mathrm{W}} Q_{z 1}-{ }^{\mathrm{W}} Q_{z 2}\right) \mathrm{s} \beta \\
\left({ }^{\mathrm{W}} Q_{x 1}-{ }^{\mathrm{W}} Q_{x 2}\right) \mathrm{s} \gamma+\left({ }^{\mathrm{W}} Q_{y 1}-{ }^{\mathrm{W}} Q_{y 2}\right) \mathrm{c} \gamma \\
\left({ }^{\mathrm{W}} Q_{x 1}-{ }^{\mathrm{W}} Q_{x 2}\right) \mathrm{s} \beta \mathrm{c} \gamma+\left({ }^{\mathrm{W}} Q_{y 1}-{ }^{\mathrm{W}} Q_{y 2}\right) \mathrm{s} \beta \mathrm{s} \gamma+\left({ }^{\mathrm{W}} Q_{z 1}-{ }^{\mathrm{W}} Q_{z 2}\right) \mathrm{c} \beta \\
0
\end{array}\right]\right\|_{\mathrm{XZ}} \\
& \left.=\left[\left({ }^{\mathrm{W}} Q_{y 1}-{ }^{\mathrm{W}} Q_{y 2}\right){ }^{2} \mathrm{~s}^{2} \gamma+\left({ }^{\mathrm{W}} Q_{z 1}-{ }^{\mathrm{W}} Q_{z 2}\right){ }^{2}\right]\right]^{1 / 2}
\end{aligned}
$$

$\mathrm{Q}_{1}$ and $\mathrm{Q}_{2}$ are on the motion trajectory of the probe upper and downward tip points, respectively. Consequently,

$$
\begin{aligned}
& \left\{\begin{array}{l}
{ }^{\mathrm{O}} Q_{y 1}={ }^{\mathrm{O}} P_{y}^{\mathrm{up}} \\
{ }^{\mathrm{o}} Q_{y 2}={ }^{\mathrm{O}} P_{y}^{\text {down }}
\end{array}\right. \\
& \Rightarrow\left\{\begin{array}{l}
\frac{L}{2} \mathrm{~s} \gamma+{ }^{\mathrm{W}} Q_{y 1} \mathrm{c} \gamma+b_{\mathrm{W}}=-\frac{h_{\mathrm{P}} \mathrm{s} \psi \mathrm{c} \theta}{2}+b_{\mathrm{P}} \\
\frac{L}{2} \mathrm{~s} \gamma+{ }^{\mathrm{W}} Q_{y 2} \mathrm{c} \gamma+b_{\mathrm{W}}=\frac{h_{\mathrm{P}} \mathrm{s} \psi \mathrm{c} \theta}{2}+b_{\mathrm{P}}
\end{array}\right. \\
& \Rightarrow\left\{\begin{array}{l}
{ }^{\mathrm{w}} Q_{y 1}=\frac{2\left(b_{\mathrm{P}}-b_{\mathrm{W}}\right)-L \mathrm{~s} \gamma-h_{\mathrm{P}} \mathrm{s} \psi \mathrm{c} \theta}{2 \mathrm{c} \gamma} \\
{ }^{\mathrm{W}} Q_{y 2}=\frac{2\left(b_{\mathrm{P}}-b_{\mathrm{W}}\right)-L \mathrm{~s} \gamma+h_{\mathrm{P}} \mathrm{s} \psi \mathrm{c} \theta}{2 \mathrm{c} \gamma}
\end{array}\right.
\end{aligned}
$$

Suppose that $D_{\mathrm{r}}$ is the real value of the pitch diameter, according to the cylindrical surface equation, the following equation can be obtained

$$
{ }^{\mathrm{W}} Q_{y i}^{2}+{ }^{\mathrm{W}} Q_{z i}^{2}=D_{\mathrm{r}}^{2} / 4, \quad i=1,2
$$

Combining the (9) and (10), we have

$$
\left\{\begin{array}{l}
{ }^{\mathrm{w}} Q_{z 1}=\sqrt{\frac{D_{\mathrm{r}}^{2}}{4}-\left[\frac{2\left(b_{\mathrm{P}}-b_{\mathrm{W}}\right)-L \mathrm{~s} \gamma-\mathrm{s} \psi \mathrm{c} \theta h_{\mathrm{P}}}{2 \mathrm{c} \gamma}\right]^{2}} \\
{ }^{\mathrm{w}} Q_{z 2}=-\sqrt{\frac{D_{\mathrm{r}}^{2}}{4}-\left[\frac{2\left(b_{\mathrm{P}}-b_{\mathrm{W}}\right)-L \mathrm{~s} \gamma+\mathrm{s} \psi \mathrm{c} \theta h_{\mathrm{P}}}{2 \mathrm{c} \gamma}\right]^{2}}
\end{array}\right.
$$

Introducing (9) and (11) into (8), the calculation result of the pitch diameter $D_{\mathrm{m}}$ can be written as: 


$$
D_{\mathrm{m}}=\left\{4 h_{\mathrm{P}}^{2} \tan ^{2} \gamma \mathrm{s}^{2} \psi \mathrm{c}^{2} \theta+\left[\left(\frac{D_{\mathrm{r}}^{2}}{4}-\left(\frac{2 b-\mathrm{s} \gamma L-\lambda}{2 \mathrm{c} \gamma}\right)^{2}\right)^{1 / 2}+\left(\frac{D_{\mathrm{r}}^{2}}{4}-\left(\frac{2 b-\mathrm{s} \gamma L+\lambda}{2 \mathrm{c} \gamma}\right)^{2}\right)^{1 / 2}\right]^{2}\right\}^{1 / 2}
$$

where $\lambda=h_{\mathrm{p}} \mathrm{s} \psi \mathrm{c} \theta, \quad b=b_{\mathrm{P}}-b_{\mathrm{W}}$ which denotes the relative deviation along the $\mathrm{Y}$ axis between the probe and the work piece in the basic coordinate system OXYZ. The system error caused by the alignment deviations is defined as follows:

$$
e=D_{\mathrm{r}}-D_{\mathrm{m}}
$$

From equations (12) and (13), the factors of alignment deviations that affect the pitch diameter result include: (1) the relative deviation $b$ along the $\mathrm{Y}$ axis between the probe and the work piece; (2) the rotation angle deviation $\gamma$ of the work piece around the $\mathrm{Z}$ axis; (3) the rotation angle deviation $\psi$ of the probe around the $\mathrm{X}$ axis and the rotation angle deviation $\theta$ of the probe around the $\mathrm{Y}$ axis. If $b=0$ and $\gamma=\psi=0$, then $D_{\mathrm{m}}=D_{\mathrm{r}}$. Meanwhile, this illustrates that the other alignment deviations do not affect the calculation result directly for the calculation method adopted in this paper.

\section{THE COMPENSATION METHOD OF ALIGNMENT CALIBRATION}

\section{A. The simplification of the model.}

There are three probe styles for various thread gauges ranging from M4 to M60 used in our measuring instrument. It is difficult and costly to measure all the alignment deviations when the probe or the fixture is fixed. These alignment deviations are typically calculated through measurement of the standard gauges and the system error can be compensated based on the alignment deviation parameters. However, during the calibration process, according to the measured value and real value of the standard gauges, it is not easy to solve the four alignment deviation parameters directly using equation (12). Some rational simplifications of equation (12) should be taken into account.

The Sobol algorithm [13, 14] of the global sensitivity analysis method is adopted to find the degree of influence of the alignment deviation parameters on the system error. According to the Sobol theory, function $f(\mathbf{x})$ can be decomposed as follows:

$$
\begin{aligned}
& f(\boldsymbol{x})=f_{0}+\sum_{i=1}^{k} f_{i}\left(x_{i}\right) \\
& \quad+\sum_{i<j} f_{i j}\left(x_{i}, x_{j}\right)+\ldots+f_{12 \ldots n}\left(x_{1}, x_{2}, \ldots, x_{n}\right)
\end{aligned}
$$

where

$$
f_{0}=\int_{\Omega} f(\boldsymbol{x}) d \boldsymbol{x},
$$

$$
\begin{gathered}
f_{i}\left(x_{i}\right)=\int f(\boldsymbol{x}) \prod_{j \neq i} d x_{j}-f_{0}, \\
f_{i j}\left(x_{i}, x_{j}\right)=\int f(x) \prod_{l \neq i, j} d x_{l}-f_{0}-f_{i}\left(x_{i}\right)-f_{j}\left(x_{j}\right)
\end{gathered}
$$

and so on. The total variance is defined as,

$$
V=\int f^{2}(\boldsymbol{x}) d \boldsymbol{x}-f_{0}^{2}
$$

and the partial variance is given by

$$
V_{i_{1}, \ldots, i_{s}}=\int f_{i_{1}, \ldots, i_{s}}^{2} d x_{i_{1}} \ldots d x_{i_{s}} .
$$

The ratios

$$
S_{i_{1}, \ldots i_{s}}=V_{i_{1}, \ldots i_{s}} / V
$$

are called the global sensitivity indices. After squaring and integrating both sides of equation (14), the following equation can be obtained

$$
1=\sum_{i=1}^{k} S_{i}+\sum_{i<j} S_{i j}+\sum_{i<j<l} S_{i j l}+\ldots+S_{1,2, \ldots, k}
$$

Introducing the total sensitivity indices in regard to parameter $x_{i}$

$$
S_{\mathrm{T}}\left(x_{i}\right)=\sum S_{(i)}
$$

where $S_{(i)}$ denotes all the sensitivity indices including parameter $i$.

In the equation (12) model, the parameters $b, \gamma, \psi$ and $\theta$ are treated as independent random variables uniformly distributed in scope $(-2 \mathrm{~mm}, 2 \mathrm{~mm}),\left(-5^{\circ}, 5^{\circ}\right),\left(-5^{\circ}, 5^{\circ}\right)$ and $\left(-5^{\circ}, 5^{\circ}\right)$. And the total sensitivity indices of each parameter are calculated: $S_{\mathrm{T}}(b)=0.9798, S_{\mathrm{T}}(\gamma)=0.3554, S_{\mathrm{T}}(\psi)=0.0017$, $S_{\mathrm{T}}(\theta)=1.460 \mathrm{e}-5$. The sensitivity indices of the parameters $\psi$ and $\theta$ are much smaller compared to the parameters $b$ and $\gamma$.

According to [15], the rotation angle deviation $\psi$ of the probe around the $\mathrm{X}$ axis can be confined in scope $\left(-1^{\circ}, 1^{\circ}\right)$ with the vision detection method. If the parameter $\psi$ is approximated to 0 , the equation (12) can be simplified as follows: 
Similarly, we have

$$
e^{\prime}=D_{\mathrm{r}}-D_{m}^{\prime}
$$

Then, the error between the simplified model (16) and the integral model (13) is

$$
E=e^{\prime}-e=D_{m}-D_{m}^{\prime}=g\left(b, \gamma, \psi, \theta, L, D_{r}\right)
$$

With the Monte Carlo method [16], the maximum model error $E_{\max }$ can be simulated with the change of thread size, which is shown in Fig.4. Since the parameters of $b, \gamma, \psi$ and $\theta$ are uniformly distributed in their scope, the random number $\xi$ from $[0,1]$ is used to generate the values. For example, $b=-2+\xi(2-(-2))=-2+4 \xi$. And the number of Monte Carlo trials $M$ is set to be $10^{6}$ according to [17]. For a settled couple of $L$ and $D_{r}$, the $E_{\max }=\max \left(E_{i}\right), i=1, \ldots, M$.

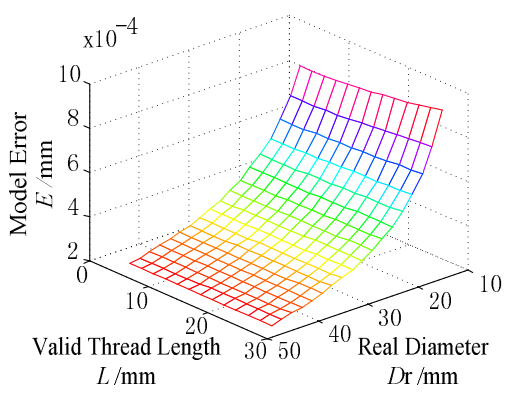

(a) $h_{\mathrm{P}}=9 \mathrm{~mm}$

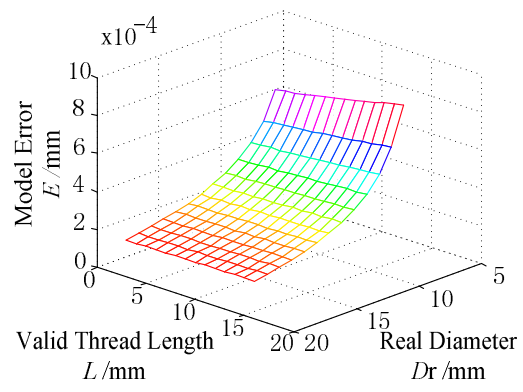

(b) $h_{\mathrm{P}}=5 \mathrm{~mm}$

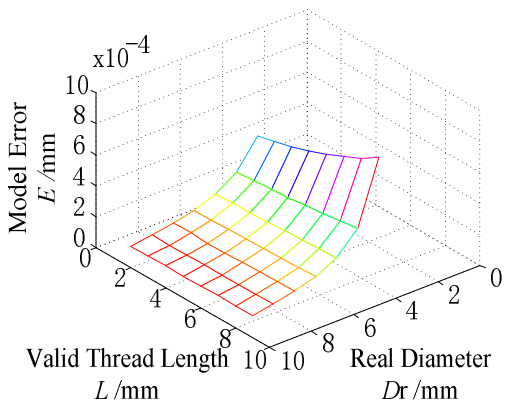

(c) $h_{\mathrm{P}}=3 \mathrm{~mm}$

Fig.4. The max model errors for three type probes.

For each probe type shown in Fig.4., the maximum model errors are less than $1 \mu \mathrm{m}$. Consequently, the simplification of substituting equation (12) with equation (16) is probable.

\section{B. Rationale for the compensation of alignment calibration.}

First, two standard gauges with different pitch diameter sizes are selected for measurement. Suppose that the valid scanning lengths of the two gauges are $L_{1}, L_{2}$ and the measuring results of the pitch diameter are $D_{\mathrm{m} 1}, D_{\mathrm{m} 2}$, respectively. Based on equation (16), the following equations can be constructed

$$
\left\{\begin{array}{l}
D_{\mathrm{m} 1}=\left(D_{\mathrm{r} 1}^{2}-\left(\frac{2 b-\mathrm{s} \gamma L_{1}}{\mathrm{c} \gamma}\right)^{2}\right)^{1 / 2} \\
D_{\mathrm{m} 2}=\left(D_{\mathrm{r} 2}^{2}-\left(\frac{2 b-\mathrm{s} \gamma L_{2}}{\mathrm{c} \gamma}\right)^{2}\right)^{1 / 2}
\end{array}\right.
$$

Solving the equations, we have

$$
\left\{\begin{array}{l}
\gamma=\arctan \frac{\sqrt{D_{\mathrm{r} 2}^{2}-D_{\mathrm{m} 2}^{2}}-\sqrt{D_{\mathrm{r} 1}^{2}-D_{\mathrm{m} 1}^{2}}}{L_{1}-L_{2}} \\
b=\frac{\sqrt{D_{\mathrm{r} 1}^{2}-D_{\mathrm{m} 1}^{2}}}{2} \cos \gamma+\frac{L_{1}}{2} \sin \gamma
\end{array}\right.
$$

Then, the thread gauge for test is detected. If the valid scanning length of the testing thread gauge is $L_{\mathrm{T}}$, the compensation formula of the real pitch diameter ${ }^{\mathrm{T}} D_{\mathrm{r}}$ is as follows:

$$
{ }^{\mathrm{T}} D_{\mathrm{r}}=\sqrt{{ }^{\mathrm{T}} D_{\mathrm{m}}^{2}+\left(\frac{2 b-\sin \gamma L_{\mathrm{T}}}{\cos \gamma}\right)^{2}}
$$

where ${ }^{\mathrm{T}} D_{m}$ denotes the measuring result of the pitch diameter for the testing thread gauge before compensation.

\section{Uncertainty analysis.}

In metrology, the measurement uncertainty is defined as a "parameter, associated with the result of a measurement, that characterizes the dispersion of the values that could reasonably be attributed to the measurand" [18]. Using equation (20), the compensation result of the pitch diameter is calculated by parameters including the alignment deviations. Therefore, the uncertainty of the measurement result is a combined standard uncertainty.

In order to simplify the expression, a variable substitution is taken. Let

$$
\begin{aligned}
\boldsymbol{X} & =\left[x_{1}, x_{2}, \ldots, x_{8}\right]^{\mathrm{T}} \\
& =\left[{ }^{\mathrm{T}} D_{\mathrm{m}}, D_{\mathrm{m} 1}, D_{\mathrm{m} 2}, D_{\mathrm{r} 1}, D_{\mathrm{r} 2}, L_{1}, L_{2}, L_{\mathrm{T}}\right]^{\mathrm{T}}
\end{aligned}
$$

and use the following equation mark to represent the combination of the (16) and (18) 


$$
{ }^{\mathrm{T}} D_{\mathrm{r}}=\Phi(\boldsymbol{X})
$$

According to the combined standard uncertainty formula [19, Sub Clause 5.2.2], one can get

$$
\begin{aligned}
& u_{c}\left({ }^{\mathrm{T}} D_{\mathrm{r}}\right)= \\
& \sqrt{\sum_{i=1}^{8}\left(\frac{\partial \Phi}{\partial x_{i}}\right)^{2} u^{2}\left(x_{i}\right)+2 \sum_{i=1}^{7} \sum_{j=i+1}^{8} \frac{\partial \Phi}{\partial x_{i}} \frac{\partial \Phi}{\partial x_{j}} u\left(x_{i}, x_{j}\right)}
\end{aligned}
$$

where $u_{c}\left({ }^{\mathrm{T}} D_{\mathrm{r}}\right)$ denotes the combined standard uncertainty of the pitch diameter result ${ }^{\mathrm{T}} D_{\mathrm{r}} ; u\left(x_{i}\right)$ is the standard uncertainty associated with the input estimate $x_{i} ; u\left(x_{i}, x_{j}\right)$ is the estimated covariance associated with $x_{i}$ and $x_{j}$. However, the variables $\boldsymbol{X}$ are independent of each other, consequently $u\left(x_{i}, x_{j}\right)=0$.

\section{COMPARISON RESULTS}

\section{A. Theoretical results.}

The comparison between single gauge and two gauges calibration is considered to be the limiting situation for alignment deviation scope, which assumes $b=-2 \mathrm{~mm}, \gamma=5^{\circ}$, $\psi=5^{\circ}$ and $\theta=5^{\circ}$, based upon the hypothesis that the diameter of the thread gauges to be measured ranges from $15 \mathrm{~mm}$ to $50 \mathrm{~mm}$ corresponding to the fixture support range. Assume the valid length $L_{\mathrm{T}}$ of the gauges to be measured varies from $8 \mathrm{~mm}$ to $30 \mathrm{~mm}$.

Fig.5.(a) shows the result of system errors for the single gauge calibration method. Each curve represents a calibrating thread gauge type. Fig.5.(b) shows the result errors for the two gauges calibration method. For the limiting situation of the alignment deviations, two gauges calibration method can reduce the system error much more than the single calibration method.

To keep the measurement accuracy, many different calibrating gauges have to be prepared for the single gauge calibration method. However, the two gauges calibration method only needs two thread gauges for calibration despite the large alignment deviations. From Fig.5.(b), the minimum value of the system errors occurs when the diameter of the measured gauge is near the diameter of the two calibrated gauges. When the diameter of the measured gauge exceeds the diameter range of the two calibrated gauges then the error increases rapidly.

In addition, the uncertainty of each test gauge can be calculated according to the equation (22). The $D_{r i}(i=1,2)$ is the real pitch diameter of the calibrated threads. In our experiment, high grade gauges are selected as the calibrated screw threads, the values and the measurement uncertainty of which are known. The uncertainty $u\left(D_{r i}\right)$ is $1 \mu \mathrm{m}$. The parameters $D_{m i}(i=1,2)$ and ${ }^{\mathrm{T}} D_{m}$ are obtained by the measurement instrument. Thus, the uncertainties $u\left(D_{m i}\right)$, $u\left({ }^{\mathrm{T}} D_{m}\right)$, which are related to the repeatability of the instrument, can be calculated according to the Bessel formula in type A uncertainty evaluation [18]. Based on the measurement data, we have $u\left(D_{m i}\right)=u\left({ }^{\mathrm{T}} D_{m}\right)=1 \mu \mathrm{m}$. The parameters $L_{1}$ and $L_{2}$ are the valid length of the threads in the $\mathrm{X}$ axis, and their uncertainty depends on the positioning repeatability in the $\mathrm{X}$ axis. The linear motor and grating ruler are adopted in the control system. After the calculation by using the Bessel formula, we have $u\left(L_{\mathrm{T}}\right)=u\left(L_{1}\right)=$ $u\left(L_{2}\right)=0.2 \mu \mathrm{m}$.

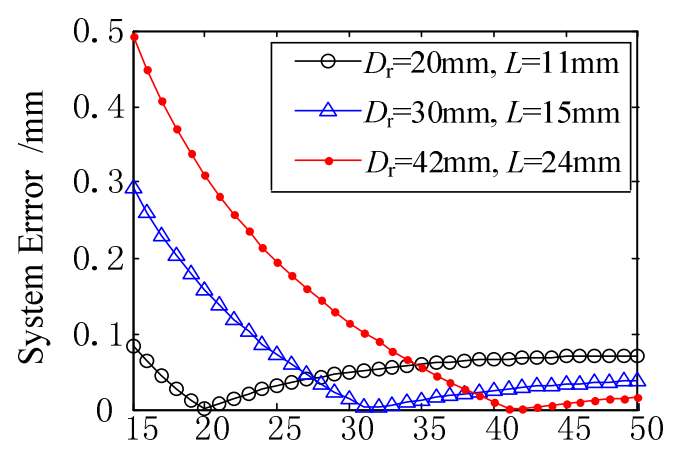

Diameter of the measured gauge $/ \mathrm{mm}$

(a) Single gauge calibration method.

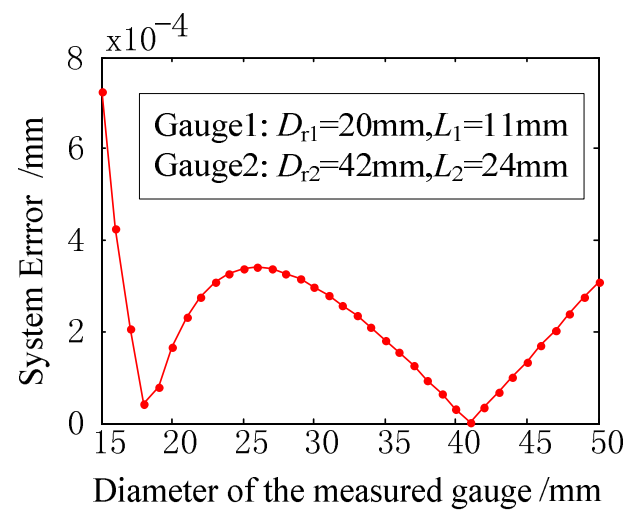

(b) Two gauges calibration method.

Fig.5. The system errors for the two calibration method in a limit situation of the alignment deviations.

The uncertainty results for the two compensation methods are shown in Fig.6. Although the uncertainty of the two gauges calibration method is a little larger than the single gauge calibration method, compared to the amount of the system error reduced by the proposed compensation method, the increasing amount of the uncertainty is much smaller.

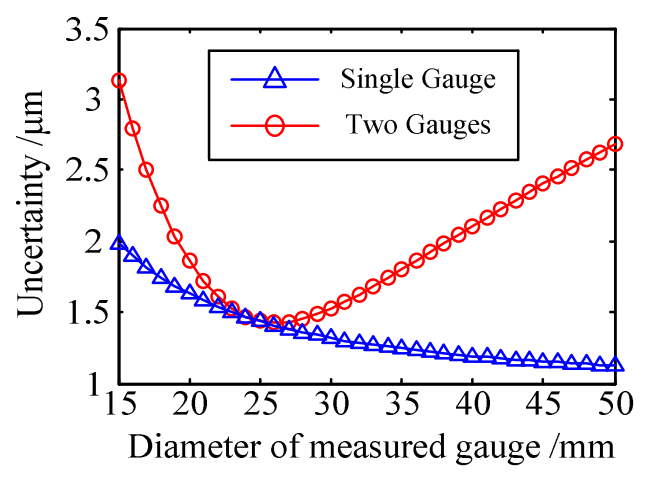

Fig.6. The uncertainty of the diameter result for both compensation methods. 


\section{B. Experimental results.}

To verify the effectiveness of the proposed compensation method, measurement experiments were carried out using thread measuring instrument, which has been developed in our laboratory. The thread measuring instrument is shown in Fig.7. The linear motor of IL12-100-A2-TR-C1 type, produced by the Kollmorgen Corporation, was chosen for the precise control. The servo driver was the SERVOSTAR-CD-5 from Panasonic Corporation. The PCI-8136 motion control card of ADLINK was adopted for the interface of the computer. The position data was monitored by the RGS20-S grating ( $50 \mathrm{~nm}$ ) of the Renishaw Corporation. The aerostatic guideway was designed to reduce the transmission error.

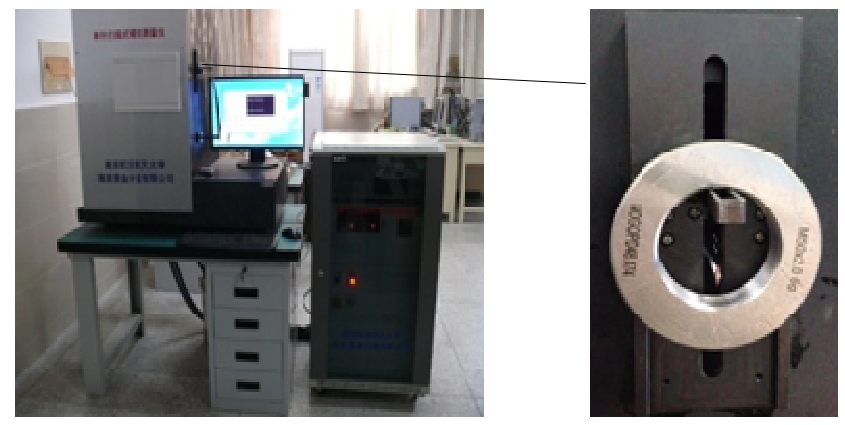

Fig.7. The thread measurement system.

First, the compensation method with single gauge calibration was tested. The thread gauge M20_6g(go) was selected as the calibrating gauge. Other thread gauges, the parameters of which are known, were measured. Measurement results for the single gauge calibration method are shown in Table 1.

Table 1. Measurement results for single gauge calibration.

\begin{tabular}{|c|l|c|}
\hline Order & \multicolumn{1}{|c|}{ Type } & Error $[\boldsymbol{\mu m}]$ \\
\hline $\begin{array}{c}\text { Calibration } \\
\text { Gauge }\end{array}$ & $\mathrm{M} 20 \times 2.5(\mathrm{go})$ & - \\
\hline 1 & $\mathrm{M} 20 \times 2.5(\mathrm{no}-\mathrm{go})$ & -4.2 \\
\hline 2 & $\mathrm{M} 42 \times 4.5(\mathrm{go})$ & +8.8 \\
\hline 3 & $\mathrm{M} 24 \times 2(\mathrm{go})$ & +0.2 \\
\hline 4 & $\mathrm{M} 24 \times 2(\mathrm{no}-\mathrm{go})$ & -3.4 \\
\hline 5 & $\mathrm{M} 16 \times 2(\mathrm{go})$ & -1.7 \\
\hline 6 & $\mathrm{M} 16 \times 2(\mathrm{no}-\mathrm{go})$ & -8.0 \\
\hline
\end{tabular}

If the difference of pitch diameter and the thread length between the calibration gauge and the test gauge are smaller, the measurement results are more accurate. Otherwise, the errors become larger. Such as the test gauge 2, the measurement error reaches to $+8.8 \mu \mathrm{m}$.

Then, the same thread gauges were measured with the compensation method proposed in this paper. The thread ring gauges $\mathrm{M} 20 \times 2.5(\mathrm{go})$ and $\mathrm{M} 42 \times 4.5$ (no-go) were selected as the two calibrating gauges. The results are shown in Table 2.
Table 2. The measurement results for the two gauges calibration.

\begin{tabular}{|c|l|c|}
\hline Order & Type & Error $[\boldsymbol{\mu m}]$ \\
\hline $\begin{array}{c}\text { Calibration } \\
\text { Gauge 1 }\end{array}$ & $\mathrm{M} 20 \times 2.5(\mathrm{go})$ & - \\
\hline $\begin{array}{c}\text { Calibration } \\
\text { Gauge 2 }\end{array}$ & $\mathrm{M} 42 \times 4.5($ no-go) & - \\
\hline 1 & $\mathrm{M} 20 \times 2.5($ no-go $)$ & +0.9 \\
\hline 2 & $\mathrm{M} 42 \times 4.5(\mathrm{go})$ & +1.2 \\
\hline 3 & $\mathrm{M} 24 \times 2(\mathrm{go})$ & +0.5 \\
\hline 4 & $\mathrm{M} 24 \times 2($ no-go $)$ & -1.5 \\
\hline 5 & $\mathrm{M} 16 \times 2($ go $)$ & +1.9 \\
\hline 6 & $\mathrm{M} 16 \times 2($ no-go $)$ & +0.9 \\
\hline
\end{tabular}

The errors for both compensation methods are compared in the histogram shown in Fig.8. From the results it can be seen that the errors obtained by the two gauges calibration method are smaller in magnitude compared to the errors due to the single gauge calibration method. This suggests that the two gauges calibration method can compensate the system error caused by alignment deviations more precisely than the single gauge calibration method.

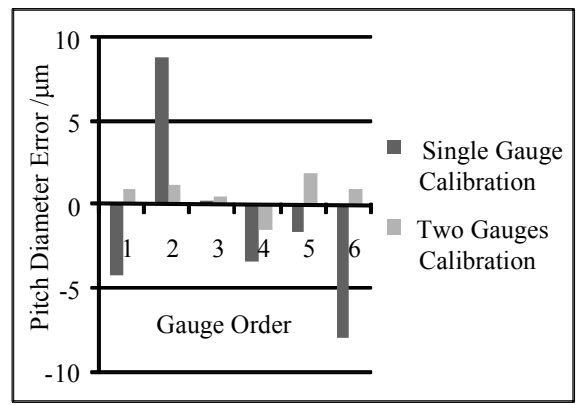

Fig.8. The measurement error of the pitch diameter.

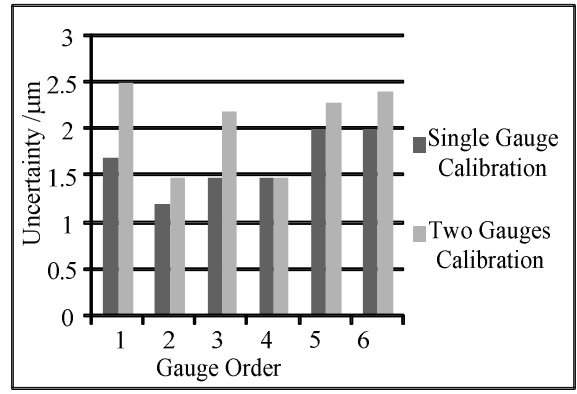

Fig.9. The uncertainty of the pitch diameter.

Fig.9. compares the uncertainty between the two compensation methods. Based on the uncertainty results of the six gauges, it can be concluded that the uncertainties for the two gauges calibration method are slightly larger than the uncertainties for the single gauge calibration method, but the system error obtained by the two gauges calibration method is much smaller, which is in accordance with the theoretical results discussed previously. The results indicate that, although the two gauges calibration method result in larger uncertainty, it makes it possible to decrease the number of the calibration gauges used and saves the calibration frequency for the continuing measurement. 
All the discussion about the result uncertainty in this paper is the combined standard uncertainty. When the expanded uncertainty is needed to express the measurement result, the coverage factor $k$ can be chosen as 2 for an interval having a level of confidence of approximately $95 \%$.

\section{CONCLUSION}

In this paper, the effect of the alignment deviations on pitch diameter of thread using the homogeneous transformation method is presented. The global sensitivity analysis is adopted to simplify the compensation equations. A compensation method with two gauges calibration is proposed. Compared to the single gauge calibration method, the advantage of the proposed method is that the system error of pitch diameter caused by the alignment deviations can be significantly reduced for the thread gauges which are supported by the corresponding fixture and probe. Measurement uncertainty analysis for the compensation formula indicates that although the two gauges calibration method results in larger uncertainty, it makes it possible to decrease the amount of the calibration gauges and save the calibration frequency for the continuing measurement.

\section{ACKNOWLEDGMENTS}

This work was sponsored by the National Natural Science Foundation of China (51175261) and supported by Nanjing Zijin Measurement Co. Ltd. The authors would like to express their sincere thanks to them.

\section{REFERENCES}

[1] Merkac, T.P., Acko, B. (2010). Comparising measuring methods of pitch diameter of thread gauges and analysis of influences on the measurement results. Measurement, 43 (3), 421-425.

[2] Zhang, H.Y., Zhang, G.X. (2008). Online test system of large screw thread. Chinese Journal of Mechanical Engineering, 44 (12), 199-202.

[3] Kosarevsky, S., Latypov, V. (2010). Development of an algorithm to detect screw threads in planar point clouds. Measurement Science Review, 10 (4), 136-141.

[4] European Association of National Metrology Institutes. (2007). Determination of pitch diameter of parallel thread gauges by mechanical probing. EURAMET/cg-10/v.01, 6-9.

[5] Hong, E., Zhang, H., Katz, R., Agapiou, J.S. (2012). Non-contact inspection of internal threads of machined parts. International Journal of Advanced Manufacturing Technology, 62 (1-4), 221-229.

[6] Huang, H.L., Jywe, W.Y., Liu, C.H., et al. (2010). Development of a novel laser-based measuring system for the thread profile of ballscrew. Optics and Lasers in Engineering, 48 (10), 1012-1018.
[7] Tong, Q., Jiao, C., Huang, H., et al. (2014). An automatic measuring method and system using laser triangulation scanning for the parameters of a screw thread. Measurement Science and Technology, 25 (3), 035202.

[8] Shchurov, I.A. (2011). Calculation of the virtual pitch thread diameter using the cloud of points from CMM. International Journal of Advanced Manufacturing Technology, 53 (1-4), 241-245.

[9] Kosarevsky, S., Latypov, V. (2013). Detection of screw threads in computed tomography 3D density fields. Measurement Science Review, 13 (6), 292-297.

[10] Galestin, R. (2006). Advanced 2D scanning: The solution for the calibration of thread ring and thread plug gauges. In 13th International Metrology Congress. Lille: French College of Metrology Press, 363-372.

[11] Galestien, R. (2003). Measurement of geometric parameters of internal and external screw thread and similar grooves. U.S. Patent No. 20030101602.

[12] Chen, G.S., Mei, X.S., Li, H.L. (2013). Geometric error modeling and compensation for large-scale grinding machine tools with multi-axes. International Journal of Advanced Manufacturing Technology, 69 (9-12), 2583-2592.

[13] Sobol, I.M., Kucherenko, S. (2009). Derivative based global sensitivity measures and their link with global sensitivity indices. Mathematics and Computers in Simulation, 79 (10), 3009-3017.

[14] Sobol, I.M., Tarantolab, S., Gatellib, D., et al. (2007). Estimating the approximation error when fixing unessential factors in global sensitivity analysis. Reliability Engineering \& System Safety, 92 (7), 957-960.

[15] Chen, S., Zhao, D.B., Lu, Y.H., Liu, K. (2013). A method for detecting alignment deviation on a thread-measuring instrument. Measurement Science and Technology, 24 (8), 085002.

[16] Rubinstein, R.Y., Kroese, D.P. (2011). Simulation and the Monte Carlo Method. John Wiley \& Sons.

[17] Joint Committee for Guides in Metrology. (2008). Evaluation of measurement data - Supplement 1 to the "Guide to the expression of uncertainty in measurement" - Propagation of distributions using Monte Carlo method, 1st edition. JCGM 101:2008.

[18] Kosarevsky, S., Latypov, V. (2013). Practical procedure for position tolerance uncertainty determination via Monte-Carlo error propagation. Measurement Science Review, 12 (1), 1-7.

[19] Joint Committee for Guides in Metrology. (2008). Evaluation of measurement data - Guide to the expression of uncertainty in measurement, 1st edition. JCGM 100:2008.

Received March 31, 2014. Accepted October 24, 2014. 